\title{
Pentavalent Sialic Acid Conjugates Block Coxsackievirus A24 Variant and Human Adenovirus Type 37-Viruses That Cause Highly Contagious Eye Infections
}

Emil Johansson, Rémi Caraballo, Nitesh Mistry, Georg Zocher, Weixing Qian, C. David Andersson, Daniel L. Hurdiss, Naresh Chandra, Rebecca Thompson, Lars Frängsmyr, Thilo Stehle, Niklas Arnberg, and Mikael Elofsson*

Cite This: ACS Chem. Biol. 2020, 15, 2683-2691

Read Online

\section{ACCESS}

山ll Metrics \& More

Article Recommendations

Supporting Information

ABSTRACT: Coxsackievirus A24 variant (CVA24v) and human adenovirus 37 (HAdV-37) are leading causative agents of the severe and highly contagious ocular infections acute hemorrhagic conjunctivitis and epidemic keratoconjunctivitis, respectively. Currently, neither vaccines nor antiviral agents are available for treating these diseases, which affect millions of individuals worldwide. CVA24v and HAdV-37 utilize sialic acid as attachment receptors facilitating entry into host cells. Previously, we and others have shown that derivatives based on sialic acid are effective in preventing HAdV-37 binding and infection of cells. Here, we designed and synthesized novel pentavalent sialic acid conjugates and studied their inhibitory effect against CVA24v and

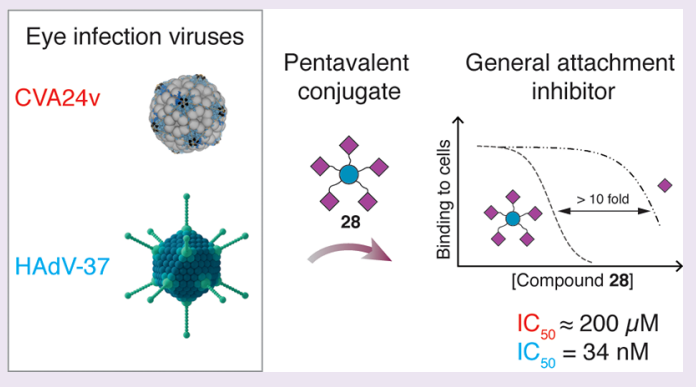
HAdV-37 binding and infection of human corneal epithelial cells. The pentavalent conjugates are the first reported inhibitors of CVA24v infection and proved efficient in blocking HAdV-37 binding. Taken together, the pentavalent conjugates presented here form a basis for the development of general inhibitors of these highly contagious ocular pathogens.

$\mathrm{E}$ very year millions of individuals around the world are affected by acute conjunctivitis. ${ }^{1,2}$ The disease can be caused by allergens, chemicals, or underlying systemic disease; however, the majority of cases is caused by viruses and bacteria. ${ }^{1-3}$ Viral conjunctivitis is estimated to cause up to $80 \%$ of all cases of acute conjunctivitis. ${ }^{1}$ Acute hemorrhagic conjunctivitis (AHC) and epidemic keratoconjunctivitis (EKC) are severe and highly contagious forms of viral conjunctivitis. ${ }^{4,5}$ AHC is characterized by a sudden onset of ocular pain, foreign body sensation, excessive lacrimation, keratitis, and conjunctivitis. ${ }^{6}$ Many of these symptoms are also shared with EKC, and it is difficult to differentiate these diseases in a clinical setting. ${ }^{7}$ The infections are generally selflimited, resolving in 1-2 weeks but can become potentially life-threatening in immunocompromised individuals., ${ }_{5,9}$ AHC spreads rapidly within communities, affecting up to $50 \%$ of the population. As a result, outbreaks can exhaust healthcare resources of affected regions and severely disrupt the economy. ${ }^{5,10}$ To date, neither antiviral agents nor vaccines are available for treating or preventing AHC or EKC.

AHC is predominantly caused by two members of the Picornaviridae family, coxsackievirus A24 variant (CVA24v) and enterovirus 70 (EV70). ${ }^{11}$ CVA24v has been reported as the principal etiological agent responsible for $>10$ million cases of AHC worldwide during the last decades, including two pandemics and numerous recurring outbreaks. ${ }^{10,12}$ To facilitate attachment and subsequent entry, members of the Picornaviridae engage a range of different cellular receptors including coxsackie and adenovirus receptor (CAR), decay accelerating factor (DAF, CD55), low density lipoprotein receptor (LDLR), human P-selectin glycoprotein ligand-1 (PSGL-1), heparan sulfate, integrins, intercellular adhesion molecule-1 (ICAM-1), and glycan-containing receptors terminating in 5- $\mathrm{N}$-acetylneuraminic acid (sialic acid). ${ }^{13-17}$ EV70 is reported to bind DAF and glycans that terminate in $\alpha 2,3$-linked sialic acid, ${ }^{13,16}$ while CVA24v binds to $\alpha 2,3-$ and $\alpha 2,6$-linked sialic acids via pentameric binding sites. ${ }^{14,15,18}$ In addition, engagement of the ICAM-1 receptor is essential for coxsackievirus A24 infection, including nonvariant and variant strains of the virus. However, the virulence and pandemic nature of the variant, AHC-causing strain seems to be linked to an enhanced ability to bind sialic acid. ${ }^{19}$ This adaptation has been suggested to enable efficient attachment of CVA24v to corneal and conjunctival cells ${ }^{15}$ and thereby enhance the transmission of viral progeny. ${ }^{19}$

Received: May 31, 2020

Accepted: August 26, 2020

Published: August 26, 2020 

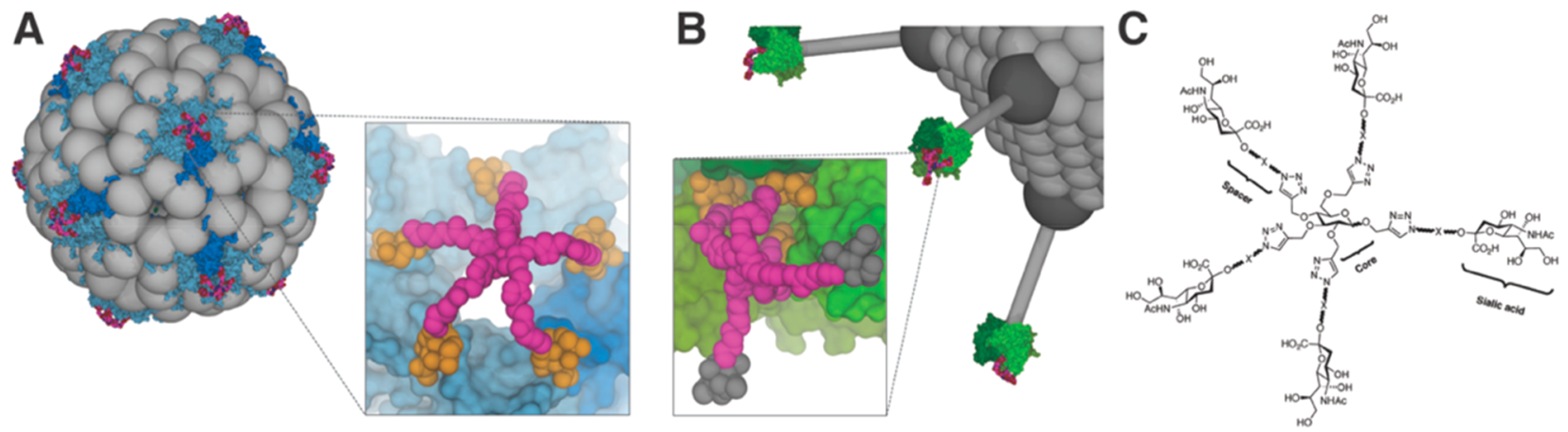

Figure 1. Design of pentavalent sialic acid conjugates. A) Schematic representation of a CVA24v particle and close-up view of one of the pentameric sialic acid binding sites with the inhibitor modeled. Inhibitor (purple) with sialic acid highlighted (yellow). B) Schematic representation of a HAdV-37 particle and zoom-in of one of the fiber knob proteins that contain trimeric sialic acid binding sites with the inhibitor modeled. Inhibitor (purple) with two sialic acids in solution (gray) and three sialic acids binding (yellow). C) Schematic design of the pentavalent sialic acid conjugates. The sialic acid residues are connected to a common core fragment via spacers of sufficient length for chelation of the pentameric binding sites of CVA24v.

\section{Scheme 1. Synthesis of the First and Second Sets of Pentavalent Sialic Acid Conjugates 25-28 ${ }^{a}$}

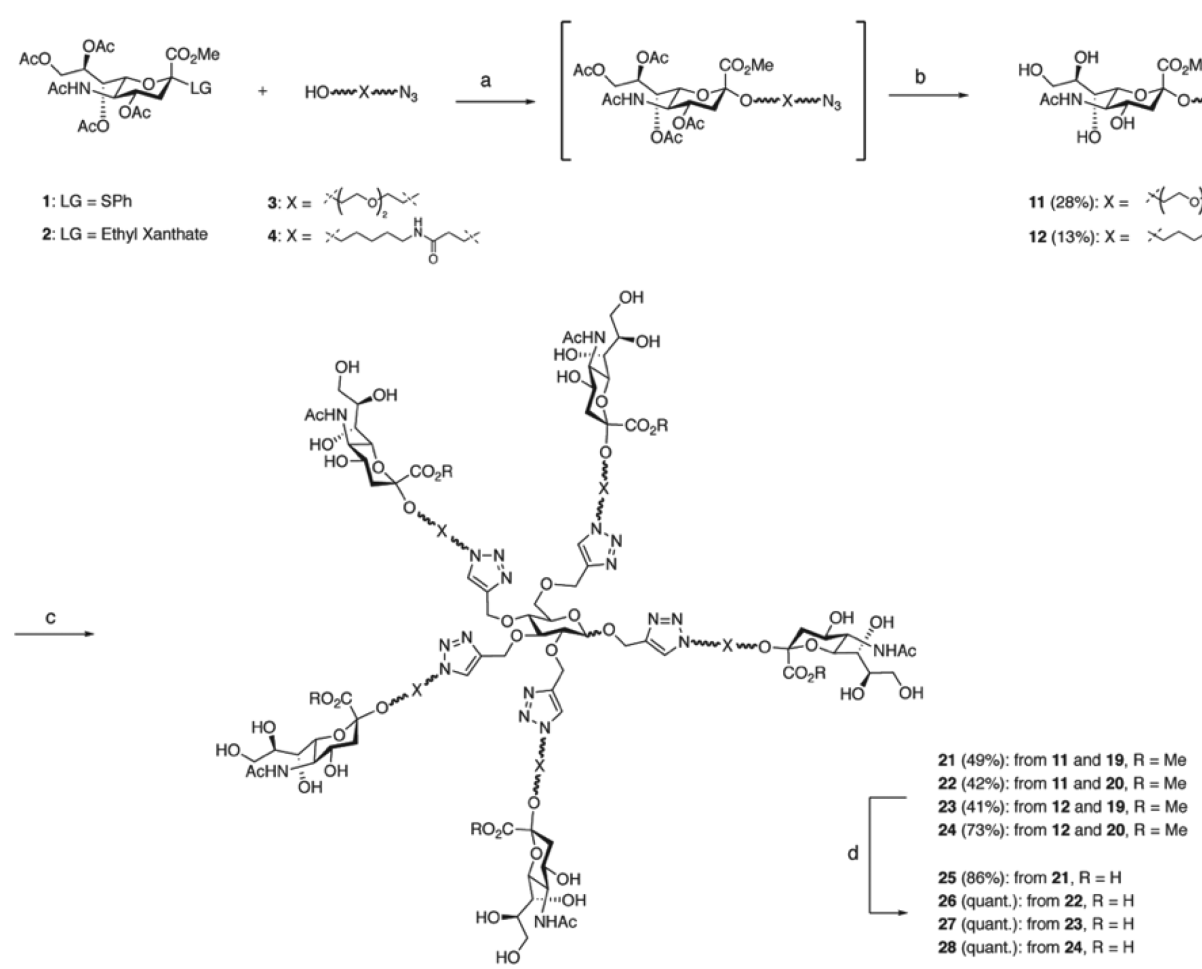

${ }^{a}$ Reagents and conditions: a) i: molecular sieves (3 $\AA$ ), 3-9, $\mathrm{CH}_{3} \mathrm{CN} / \mathrm{CH}_{2} \mathrm{Cl}_{2}(2: 1)$, rt, 30 min, ii: silver trifluoromethanesulfonate, $\mathrm{IBr},-74{ }^{\circ} \mathrm{C}$, $5.5 \mathrm{~h}$. b) Sodium methoxide, methanol, rt, $3 \mathrm{~h}$, ii: $\mathrm{H}^{+}$ion exchange resin. c) $\mathrm{CuSO}_{4} \cdot 5 \mathrm{H}_{2} \mathrm{O}$, sodium ascorbate, tetrahydrofuran/ $\mathrm{H}_{2} \mathrm{O}(1: 1), 19$ or 20 , $50{ }^{\circ} \mathrm{C}, 5 \mathrm{~h} \rightarrow \mathrm{rt}, 18$ h. d) i: $\mathrm{LiOH}$, methanol, rt, 24 h, ii: $\mathrm{H}^{+}$ion exchange resin.

EKC is contracted by millions of individuals worldwide every year. $^{20}$ The disease is caused by members of the Adenoviridae family (HAdV-8, -19, -37, -53, -56, and -64), with HAdV-37 serving as a leading causative agent. ${ }^{21}$ EKC-causing human adenoviruses are reported to engage CD46 or CAR and $\alpha 2,3$-linked sialic acids to facilitate attachment. ${ }^{22,23}$ More recently, it was established that HAdV-37 binds to glycoproteins containing the GDla glycan motif via its homotrimeric fiber knob proteins. ${ }^{21}$

Sialic acid is a highly polar and negatively charged ninecarbon monosaccharide. It is abundantly expressed on the surface of all cell types and serves important roles in mediating or modulating a variety of physiological and pathophysiological processes. ${ }^{24}$ For a number of diseases, it has been proposed that sialic acid conjugates can be used for therapeutic intervention. However, in general terms, development of carbohydrate-based therapeutics is challenging due to poor pharmacological properties imparted by their high polarity, metabolic vulnerability, and low affinity protein-carbohydrate interactions. In addition, preparation of carbohydrates can be both cumbersome and expensive. ${ }^{25}$ In the case of CVA24v and HAdV-37, many of these downsides can potentially be circumvented by the design of multivalent ligands ${ }^{26}$ intended for topical administration, e.g., eye drops, creams, or ointments. The design of multivalent carbohydrate ligands offers an excellent strategy to achieve high avidity binding to 
A

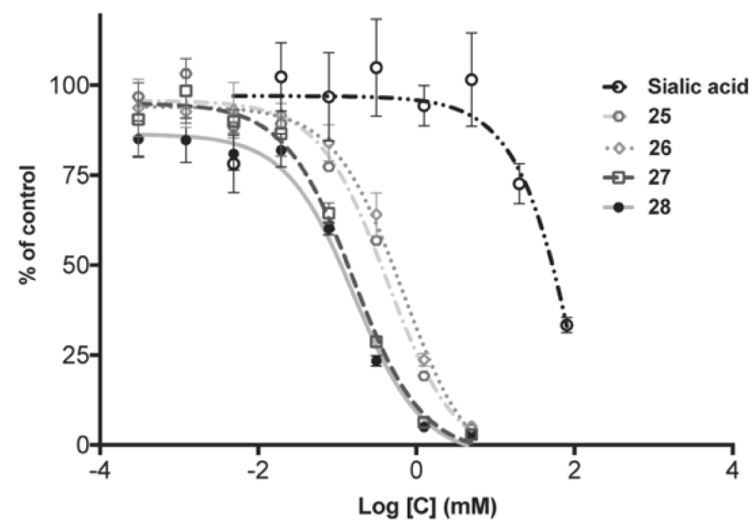

B

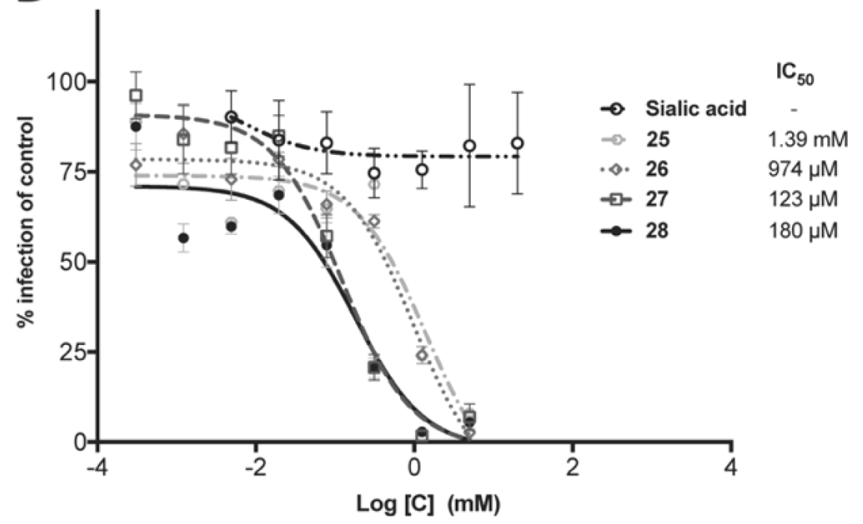

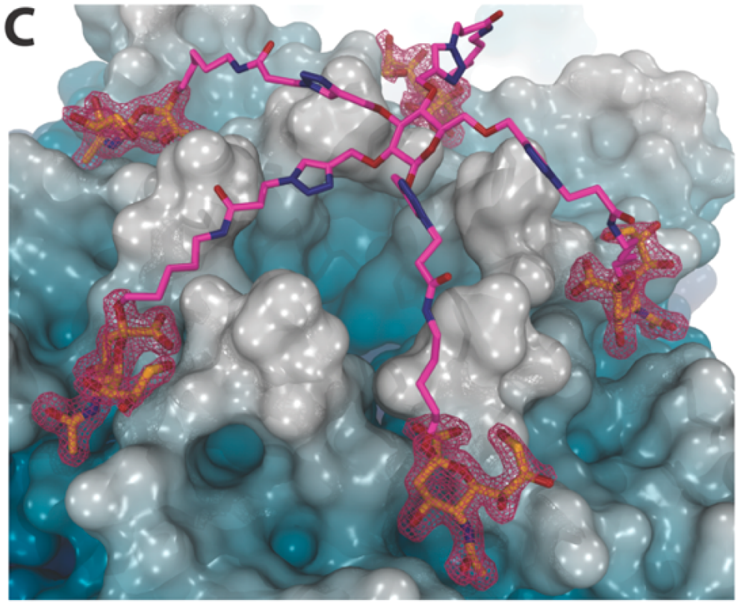

D

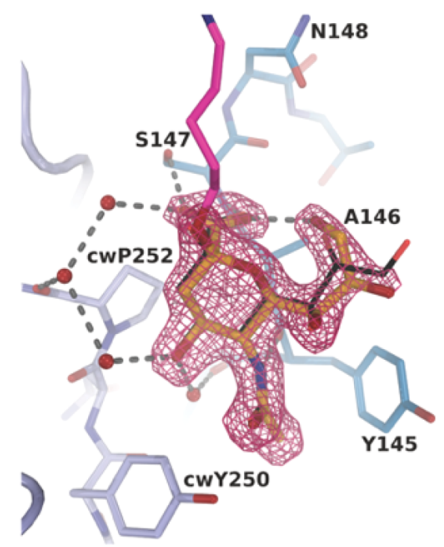

Figure 2. Effect of pentavalent sialic acid conjugates on CVA24v binding and infection of HCE cells and binding of 28 to CVA24v penton. A) Virion binding in the presence of inhibitors at different concentrations at $4{ }^{\circ} \mathrm{C}$. B) Infection at different concentrations of inhibitors at $4{ }^{\circ} \mathrm{C}$. Error bars are shown as the standard error of the mean (SEM). Data are presented as the \% of control that is the value obtained in the absence of an inhibitor. All experiments were performed in duplicate and for a minimum of two times. C) Structure of the 28-CVA24v complex. The observed electron density is shown in the cage; the remaining parts of $\mathbf{2 8}$ have been modeled for clarity. D) Interactions of the sialic acid moiety of $\mathbf{2 8}$ to amino acids in the ligand binding site of CVA24v.

lectins ${ }^{26-28}$ via the "cluster glycoside effect" ${ }^{27}$ or proximity/ statistical effects. $^{28}$ The cluster effect is caused by the binding of several monosaccharide ligands to several binding sites on the lectin, while the proximity/statistical effect is caused by a slower off-rate of a multivalent ligand in comparison to a monovalent ligand based on reasoning of proximity. ${ }^{28}$ Pivotal work with Shiga and cholera toxins demonstrated that multivalent carbohydrate ligands tailored to lectin valency and topology produce dramatic enhancements in avidity. ${ }^{29,30}$ The bacterial $\mathrm{AB}_{5}$ Shiga toxin and radially symmetric multivalent glycoconjugates were further used to develop and validate a quantitative model to analyze thermodynamic parameters of multivalent interactions. ${ }^{31}$

To date, the only substance reported to inhibit attachment of CVA24v to cells is a 13 -valent sialic acid human serum albumin conjugate. ${ }^{15}$ For HAdV-37, we and others have shown that trivalent and multivalent sialic acid conjugates are potent inhibitors of HAdV-37 attachment and infection. ${ }^{32-34}$ In this study, we developed novel pentavalent sialic acid conjugates with the potential to act as general inhibitors of both AHC and EKC. Such inhibitors could be applied via topical administration and used for treatment and prophylactics to alleviate experienced symptoms and to limit transmission of these highly contagious infections.

\section{RESULTS AND DISCUSSION}

Design of Pentavalent Sialic Acid Conjugates. We previously reported that trivalent sialic acid conjugates efficiently inhibit HAdV-37 infection of human corneal epithelial (HCE) cells. ${ }^{32,33}$ These inhibitors were designed to chelate the sialic acid binding sites, ${ }^{33}$ which are arranged in a symmetric trimeric fashion in the knob domain of the HAdV37 twelve fibers protruding from the virus particle. We reasoned that a similar strategy could be applied to CVA24v. In contrast to HAdV-37, CVA24v has five sialic acid binding sites organized over each of the twelve relatively flat surfaces of the virus particle. ${ }^{18}$ Thus, we hypothesized that the sialic acid binding sites of CVA24v could effectively be chelated by pentavalent sialic acid conjugates (Figure 1A). Such pentavalent inhibitors would also have the potential to act on HAdV-37 by providing a high local concentration of sialic acid upon binding to the virion by engaging one to three of the sialic acid binding sites of the fiber knob protein with the nonengaged sialic acids in close proximity (Figure 1B).

Perpropargylated D-glucose was selected as the core fragment (Figure 1C), ${ }^{35}$ as it allows rapid access to pentavalent sialic acid conjugates via "click" chemistry. Further, glucosides can be accessed in either $\alpha$ - or $\beta$-configuration opening an 
interesting opportunity to study potential differences in binding mode and potency between the two anomers. Two spacer fragments (a polyethylene glycol (PEG) fragment and an amide-containing alkyl fragment) were set to contain an azide function in one end and a hydroxyl group in the other. The length of these spacers can be tuned to study the effect of spacer length on potency. ${ }^{32,33,36}$ The design was validated by molecular modeling of pentavalent conjugates 25-28 (Scheme 1) to CVA24v (Figure S1). The resulting complexes were subjected to conformational searches followed by energy minimizations of the spacers. The final conformations were evaluated, no steric clashes between the conjugates and CVA24v were evident, and the carbon-carbon bond lengths and angles showed no strain and were in agreement with benchmark calculations (Table S1). Geometries for the $\alpha$ - and $\beta$-configuration of the core were similar to slightly less strain on the latter.

Synthesis of the First Set of Pentavalent Conjugates. Synthesis of the pentavalent sialic acid conjugates 25-28 was achieved in four steps from the sialic acid donors $\mathbf{1}^{37}$ and $2^{37}$ (Scheme 1, SI). The core fragment $19(\alpha$-core $)$ was synthesized by a two-step procedure involving microwaveaccelerated Fischer glycosylation of D-glucose using propargyl alcohol, $^{38}$ followed by $O$-alkylation ${ }^{35}$ with an overall yield of $23 \%$. The spacer fragment 3 was synthesized in $95 \%$ yield by substituting the corresponding alkyl chloride with an azide using $\mathrm{NaN}_{3}$. Spacer fragment 4 was isolated in $62 \%$ yield by coupling 5-amino-1-pentanol with 3-bromopropionyl chloride, followed by azide formation. Donor 1 was sialidated using spacer fragments 3 and 4, respectively, under promotion of silver trifluoromethanesulfonate and $\mathrm{IBr}^{39}$ affording the corresponding azido acetyl protected sialosides. Subsequent $O$-deacetylation afforded the $\alpha$-anomerically pure azido sialosides 11 and 12, respectively, in $28 \%$ and $13 \%$ yield over two steps. Compounds $\mathbf{1 1}$ and $\mathbf{1 2}$ were then "clicked" to the pentapropargylated glucose cores $\mathbf{1 9}$ and $\mathbf{2 0}^{35}$ affording the corresponding pentavalent sialic acid methyl esters 21-24 in $41-73 \%$ yields. Subsequent saponification provided the corresponding pentavalent sialic acid conjugates 25-28 in $86 \%$ to quantitative yields after neutralization.

Pentavalent Sialic Acid Conjugates Inhibit CVA24v Attachment to HCE Cells. To evaluate the biological effect of the pentavalent conjugates, binding inhibition assays were performed. ${ }^{15}$ Compared with untreated virions, the attachment of ${ }^{35}$ S-labeled CVA24v whole virus particles to HCE cells was significantly attenuated when preincubated with 25-28 (Figure 2A). All inhibitors were significantly more potent than sialic acid $\left(\mathrm{IC}_{50} \gg 2 \mathrm{mM}\right)$ and disialyllacto- $N$-tetraose (50\% reduction in binding at $2 \mathrm{mM}){ }^{18}$ Inhibitors 27 and 28 both containing an amide spacer were equally potent $\left(\mathrm{IC}_{50} 159\right.$ $\mu \mathrm{M}$ and $163 \mu \mathrm{M}$, respectively), while the PEG based inhibitors 25 and 26 showed lower potency $\left(\mathrm{IC}_{50} 423 \mu \mathrm{M}\right.$ and $670 \mu \mathrm{M}$, respectively). Thus, the inhibitors containing an amide spacer were 3- to 4-fold more potent than inhibitors containing a PEG spacer. All inhibitors showed similar absolute potency to the previously reported 13 -valent sialic acid conjugate. ${ }^{15}$ However, in terms of relative ligand efficiency, the ratio of the affinity of a ligand divided by the number of heavy atoms in the molecule, 25-28 were significantly more efficient at inhibiting CVA24v attachment.

Pentavalent Sialic Acid Conjugates Inhibit CVA24v Infection. To further evaluate the biological effect of the pentavalent conjugates, infection assays were performed. ${ }^{32}$ All four pentavalent conjugates 25-28 inhibited CVA24v virus particles from infecting HCE cells (Figure 2B), thus confirming the trends from the binding assays. Compounds 27-28 were 5- to 11-fold more potent than 25-26. All inhibitors were significantly more efficient than sialic acid monosaccharide that showed no apparent effect on infection at $4{ }^{\circ} \mathrm{C}$ (Figure 2B).

Increasing the temperature to physiological conditions (37 $\left.{ }^{\circ} \mathrm{C}\right)$ did not significantly alter the effect of the inhibitors on the infection (Figure S2), thus indicating the inhibitors are efficient despite the decreased utilization of sialic acid at increasing temperature by CVA24v. ${ }^{19}$

X-ray Crystallography Supports Binding of the Pentavalent Sialic Acid Conjugates to CVA24v. Next, we investigated the binding modes of 25-28 by X-ray crystallography. Structures of all four (25-28) inhibitorCVA24v complexes were established with resolutions up to 1.8 A. All structures showed clear electron density for the sialic acid in all of the five binding sites, in addition to a couple of the closest spacer atoms. The remaining parts of the inhibitors were not detected, likely due to high flexibility and adaptation of several conformations; these have been modeled into the 28-CVA24v complex (Figure 2C). The binding of 25-28 is very similar to the binding observed for other glycan-CVA24v structures with most of the contacts retained (Figure 2D). ${ }^{18}$ The key contributors to inhibitor binding are Ser147, Tyr250, and Tyr145 that directly interact with the sialic acid units of the inhibitors. Ser147 interacts with the carboxylate group of sialic acid by donating hydrogen bonds from its side chain hydroxyl group and its main chain amide proton. The side chain of Tyr250 donates a hydrogen bond and a watermediated hydrogen bond to the C5-acetamido carbonyl oxygen and the $\mathrm{C} 4$ hydroxyl group of sialic acid, respectively. The main chain amido carbonyl of Tyr 145 accepts a hydrogen bond from the $\mathrm{C} 5$ acetamido- $\mathrm{NH}$ group of sialic acid. Moreover, a water-mediated hydrogen bond is contributed by the carbonyl oxygen of Pro252. To stabilize the conformation of Tyr145, an extensive $\pi-\pi$ stacking network involving Tyr145, Arg254, and Phe95 appears important for enabling contacts to the sialic acid unit. Hydrophobic contacts are also contributed by Tyr145, Ala146, and Pro252 nonpolar regions of the binding site. The C9 hydroxyl of the sialic acid entity of $\mathbf{2 8}$ engages in intramolecular hydrogen bonding to the carboxylate group causing the glycerol chain to adopt a conformation that previously was observed only as a minor conformation for other glycan-containing structures 4Q4X and 4Q4Y (Figure 2D). ${ }^{18}$ On the basis of the structural data, an explanation to this peculiar interaction could not be determined. The absence of electron density for the core and spacer fragments of $\mathbf{2 5 - 2 8}$ is in line with previous observations with trivalent sialic acid conjugates containing long and flexible spacer fragments. ${ }^{32}$ In that case, successive shortening of the spacers resulted in decreased flexibility and increased potency, eventually resulting in detection of density for entire molecules including spacers and core. ${ }^{33}$

Design and Synthesis of the Second Set of Pentavalent Sialic Acid Conjugates. The second set of compounds focused on studying the effect of spacer length on potency. Due to their higher potency, inhibitors containing an amide appeared more attractive for probing of a tailor-made chelation of the sialic acid binding sites. Thus, compounds 39-42 containing successively shorter spacers and 43-46 containing longer spacers were designed (Figure 3A). Place- 

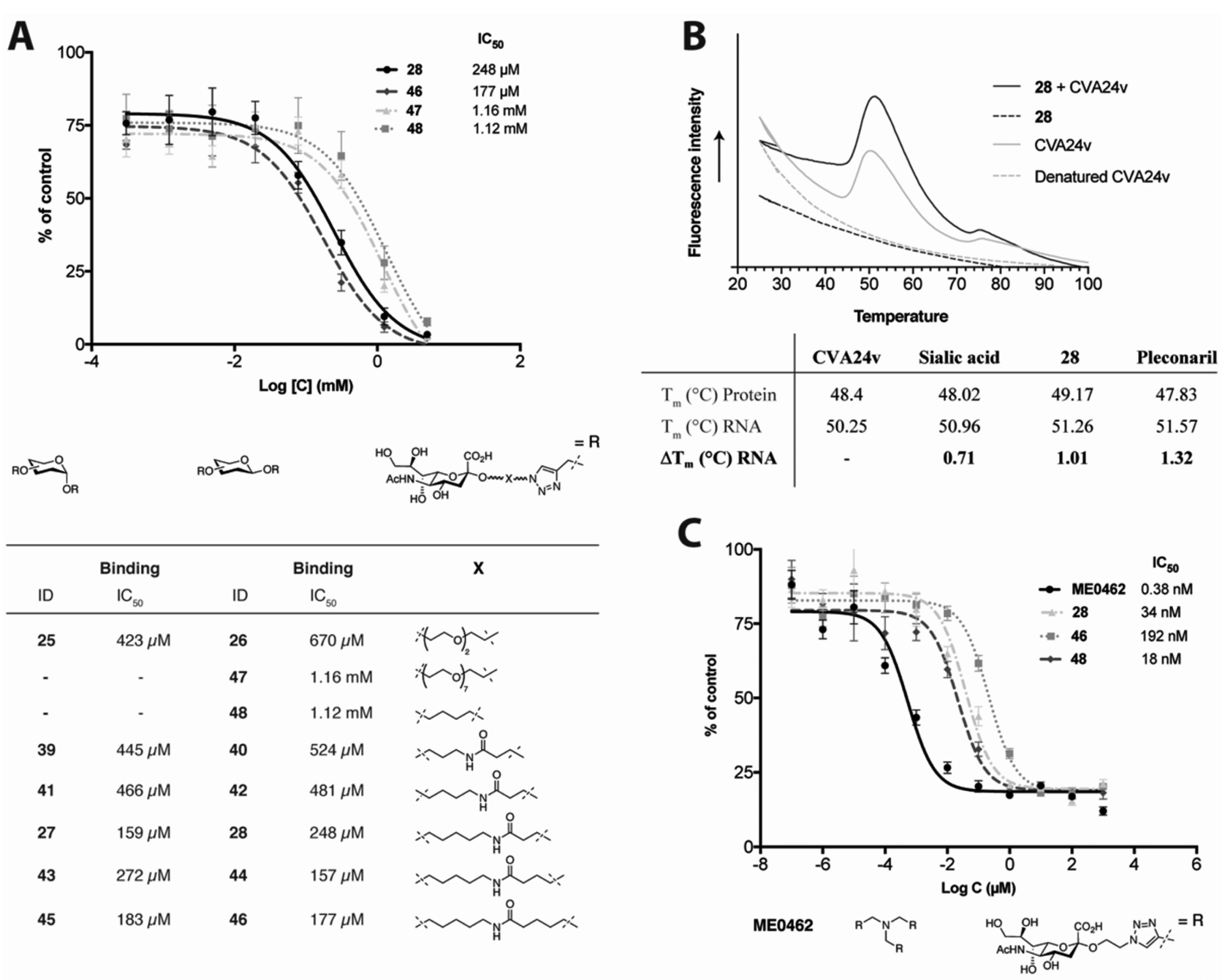

\begin{tabular}{ccccl}
\hline $\mathrm{ID}$ & $\begin{array}{c}\text { Binding } \\
\mathrm{IC}_{50}\end{array}$ & $\mathrm{ID}$ & $\begin{array}{c}\text { Binding } \\
\mathrm{IC}_{50}\end{array}$ \\
\hline 25 & $423 \mu \mathrm{M}$ & 26 & $670 \mu \mathrm{M}$ \\
- & - & 47 & $1.16 \mathrm{mM}$ \\
- & - & 48 & $1.12 \mathrm{mM}$ \\
39 & $445 \mu \mathrm{M}$ & 40 & $524 \mu \mathrm{M}$ \\
41 & $466 \mu \mathrm{M}$ & 42 & $481 \mu \mathrm{M}$ \\
43 & $159 \mu \mathrm{M}$ & 28 & $248 \mu \mathrm{M}$ \\
45 & $272 \mu \mathrm{M}$ & 44 & $157 \mu \mathrm{M}$
\end{tabular}

Figure 3. Effect of spacer length on CVA24v and HAd-V37 binding to HCE cells and effect of 28 on the thermal stability of CVA24v. A) CVA24v binding at $4{ }^{\circ} \mathrm{C}$ in the presence of inhibitors at different concentrations. For clarity, only representative examples from the series of spacer optimized compounds are illustrated in the diagram. Binding inhibition data for all pentavalent sialic acid conjugates 25-28 and 39-48 are summarized in the table, and their corresponding curves can be found in Figure S3. Error bars are shown as SEM. Data are presented as the \% of control that is the value obtained in the absence of an inhibitor. All experiments were performed in duplicate and for a minimum of two times. B) Effect of $\mathbf{2 8}$ on thermal stability of CVA24v. Fluorescence curves of CVA24v with (solid black line) and without (solid gray line) 28. The dashed lines correspond to fluorescence of $\mathbf{2 8}$ in the absence of CVA24v (black) and fluorescence of denatured CVA24v (gray line). The table below the graph shows a summary of CVA24v $T_{\mathrm{m}}$ and $T_{\mathrm{m}}$ shifts $\left(\Delta T_{\mathrm{m}}\right)$ as measured for protein (measured by SYPRO orange) and RNA with and without treatment of $100 \mu \mathrm{M}$ sialic acid, pleconaril, or 28 . All graphs are presented in the SI. C) HAd-V37 binding at $4{ }^{\circ} \mathrm{C}$ in the presence or absence of inhibitors at different concentrations. Only representative examples from the complete series of spacer optimized pentavalent conjugates were tested, in addition to trivalent sialic acid conjugate $\mathbf{M E 0 4 6 2}{ }^{33}$ used as a control. Error bars in A and C are shown as SEM. Data are presented as the $\%$ of control that is the value obtained in the absence of an inhibitor. All experiments were performed in duplicate and for a minimum of two times.

ment of the amide was probed by moving it closer to the sialic acid residues in compounds 39-42.

Compounds 40 and 46 , containing the shortest and longest amide-containing spacers, respectively, were subjected for further evaluation using computational methods (Figure S3 and Table S1). In an analogous manner to the first set, modeling of 40 and 46 to CVA24v, including conformational searches and energy minimizations, indicated that the spacers were of sufficient length to not cause strain on the spacers, nor were steric clashes with the amino acid backbone of the virion evident (Table S1 and Figure S3, respectively). Thus, the designed compounds 39-46 could be used to probe for the optimal length required for a tailor-made chelation of the binding sites.

To challenge the spacer length, 47 and 48 were designed (Figure 3A). Modeling of $\mathbf{4 8}$ to CVA24v showed severe strain, manifested by a large average $\mathrm{C}-\mathrm{C}-\mathrm{C}$ bond angle of $123.3^{\circ}$ (Figure S3 and Table S1). In addition, steric clashes occurred between the aliphatic spacers of $\mathbf{4 8}$ and amino acid residues of the virion thus indicating that, although possible, simultaneous binding of all five sialic acid residues to the pentameric binding sites of CVA24v is associated with significant energy costs. Therefore, the potency of $\mathbf{4 8}$ should be significantly decreased as the length of its spacers is unlikely to be of optimal length for chelating the sialic acid binding sites. In contrast, 47 is unlikely to act as an optimal chelator due to its extended length, and its potency should, therefore, be reduced as binding is likely associated with large entropic costs due to significant loss of translational and rotational degrees of freedom. $^{36}$

Synthesis of pentavalent conjugates 39-48 was achieved in an analogous manner to the synthesis of 25-28, with minor 
modifications (SI). The use of donor 2 in combination with decreased concentration of spacer fragment 4 proved beneficial in the synthesis of azido sialoside 12, improving the yield to $35 \%$ over two steps. Therefore, donor 2 was used in the synthesis of azido sialosides 13-18.

Spacer Length of the Conjugates Affects the Inhibitory Potency against CVA24v. The inhibitory effect of the second set of compounds was evaluated in cell binding assays. The most potent compound $44\left(\mathrm{IC}_{50} 157 \mu \mathrm{M}\right)$ was measured to be 1.6-fold more potent than reference compound $28\left(\mathrm{IC}_{50} 248 \mu \mathrm{M}\right)$ (Figure 3A). As expected, $47\left(\mathrm{IC}_{50} 1.16\right.$ $\mathrm{mM})$ and $48\left(\mathrm{IC}_{50} 1.12 \mathrm{mM}\right)$ with very long or short spacers were the least efficient of the series proving 5.4- and 4.6-fold less potent than 28, respectively (Figure 3A). Compounds 3942, containing shorter spacers, were 1.8 - to 2.1 -fold less potent than 28 , while compounds $43-46$, containing longer spacers, were equally or 1.4 - to 1.6 -fold more potent than 28 (Figure $3 \mathrm{~A}$ and Figure S3 for dose-response curves). Thus, increased spacer length resulted in slightly improved potency. Correlation between anomeric configuration of the core and potency could, however, not be demonstrated. This can potentially be attributed to the absence of direct contacts between the protein backbone of the virion and the spacers and core. Further, the compounds are able to bind with either the $\alpha$ - or the $\beta$-face of the core projecting toward the virion thus rendering potential effects of core configuration to a minimum. However, it is possible that the anomeric configuration might play a critical role in combination with a certain linker type and length. On the other hand, a weak correlation between spacer length and potency was observed as compounds with longer spacers were 3 - to 4 -fold more potent than compounds with shorter spacers. However, greatly increasing the length of the spacer was not beneficial, reflecting the greater entropic costs of binding of a more flexible ligand. Spacer type did not seem to affect potency as compounds 25 and 26 containing a PEG spacer were equally potent to compounds 41 and 42 containing an amide spacer of comparable length (Figure 3A).

Crystal structures of all inhibitor-virus complexes were obtained and resolved as previously described; however, similar to the 28-CVA24v complex (pdb code 6TSD), density was only observed for the sialic acid units. Thus, decreasing the flexibility of the conjugates by decreasing the spacer length of 39-42 did not lead to detection of additional electron density from the spacer(s) or core(s).

Pentavalent Sialic Acid Conjugates Provide Mild Stabilization of the CVA24v Particle. Antiviral drugs targeting the viral capsid have been suggested to act by stabilization of the virus particle, ${ }^{40}$ thereby preventing (or delaying) receptor mediated conformational changes required for uncoating. ${ }^{41}$ Pleconaril is one of the most studied capsidbinding molecules and is known to bind to the hydrophobic pocket of most Enteroviruses where it replaces the hydrophobic pocket factor (a fatty acid), which stabilizes the virus particle preventing uncoating. ${ }^{42,43}$ Contrarily, capsid-binding molecules could also act by destabilizing the virus particle triggering conformational changes, resulting in early uncoating and inactivation of the virus. To study the effect of the inhibitors on the stability of the CVA24v particle, we performed the Particle Stability Thermal Release Assay (PaSTRy). ${ }^{44}$ We selected the original inhibitor $\mathbf{2 8}$ as a representative example of the synthesized compounds. In addition, we selected pleconaril and sialic acid monosaccharide as controls. The melting temperature $\left(T_{\mathrm{m}}\right)$ of the CVA24v particle was calculated to be $48.40{ }^{\circ} \mathrm{C}$ for the proteins $\left(T_{\mathrm{m}}\right.$ protein) and $50.25{ }^{\circ} \mathrm{C}$ for release of the viral genome ( $T_{\mathrm{m}}$ RNA) (Figure $\left.3 \mathrm{~B}\right)$. The melting profile is in the same range as for other known Enteroviruses. ${ }^{41}$ Treatment of CVA24v with the sialic acid monosaccharide shifted the release of the viral genome to $50.96{ }^{\circ} \mathrm{C}$ indicating mild thermal stabilization (Figure S4). For the pleconaril control, the melting temperature shifted to 51.57 ${ }^{\circ} \mathrm{C}$ (Figure S4), which is a relatively weak effect in comparison to other known Enteroviruses where pleconaril had a thermal stabilizing effect of $3-6{ }^{\circ} \mathrm{C}$. ${ }^{41}$ Similar to pleconaril and sialic acid, compound 28 showed a mild stabilizing effect by shifting the $T_{\mathrm{m}}$ for release of the viral genome to $51.26^{\circ} \mathrm{C}$ (Figure 3B). This indicates that the mechanism of inhibition does not involve destabilization of the viral capsid, and early release of the viral genome, upon compound binding.

Pentavalent Sialic Acid Conjugates Inhibit HAdV-37 Binding to HCE Cells. To study the ability of the pentavalent conjugates to act as general inhibitors of $\mathrm{AHC}$ and $\mathrm{EKC}$, we performed binding experiments using HAdV-37 and HCE cells. ${ }^{23}$ Compared with untreated virions, the attachment of ${ }^{35}$ S-labeled HAdV-37 to HCE cells was significantly reduced when preincubated with 28, 46, and 48 (Figure 3C). All tested compounds were significantly more potent than the sialic acid monosaccharide $\left(\mathrm{IC}_{50} 1.2 \mathrm{mM}\right)^{33}$ and the GDla glycan $\left(\mathrm{IC}_{50}\right.$ $90.8 \mu \mathrm{M}){ }^{21}$ Compound $48\left(\mathrm{IC}_{50} 18 \mathrm{nM}\right)$ was roughly 3.7 orders of magnitude more potent than GDla, while 28 ( IC $_{50}$ $34 \mathrm{nM})$ and 46 ( $\left.\mathrm{IC}_{50} 192 \mathrm{nM}\right)$ proved about 2- and 11-fold less effective than 48, respectively. ${ }^{21}$ These results were in agreement with previous observations that multivalent sialic acid conjugates with shorter spacers are more potent against HAdV-37. ${ }^{32,33}$ The pentavalent conjugates were, however, not as efficient at inhibiting $\mathrm{HAdV}-37$ binding as reference compound ME0462 ( $\left.\mathrm{IC}_{50} 0.38 \mathrm{nM}\right){ }^{33}$ ME0462 has a nearly perfect fit in the fiber knob, and our previous data indicate that increasing valency might not improve avidity as suggested by theoretical models. ${ }^{31}$ The relatively modest avidity gains obtained against CVA24v are likely the result of CVA24v engaging multiple receptors on the host cells. ${ }^{15,19}$ Nevertheless, compounds 46 and $28\left(\mathrm{IC}_{50} 177 \mu \mathrm{M}\right.$ and $248 \mu \mathrm{M}$ against CVA24v, respectively) proved very efficient at inhibiting HAdV-37 supporting the prospects of developing a general inhibitor against two of the major causes of EKC and AHC.

The potency of the compounds toward CVA24v may need further improvement. However, administration is likely possible in millimolar concentrations without adverse side effects since sialic acid is nontoxic and abundantly expressed throughout the human body. Further, repeated administration of ME0462 in high doses to rabbits did not result in any toxic reactions or adverse side effects. ${ }^{33}$

Spacer Length of Pentavalent Conjugates Affects Binding to CVA24v and HAdV-37. The mechanism of potency enhancements in multivalent ligand systems is suggested to occur via chelation, statistical rebinding, crosslinking, and aggregation or via a combination of these mechanisms. An internal diffusion (bind and slide) mechanism has also been proposed for large systems containing many (sub)ligands. ${ }^{28}$ We were interested to study if the mechanism of potency enhancement occurred via cross-linking and aggregation. To study this, we performed negative staining electron microscopy experiments with CVA24v and HAdV-37 (Figure 4). Compounds 28, 46, and 48 were selected from the set of inhibitors as they present a compromise between spacer 


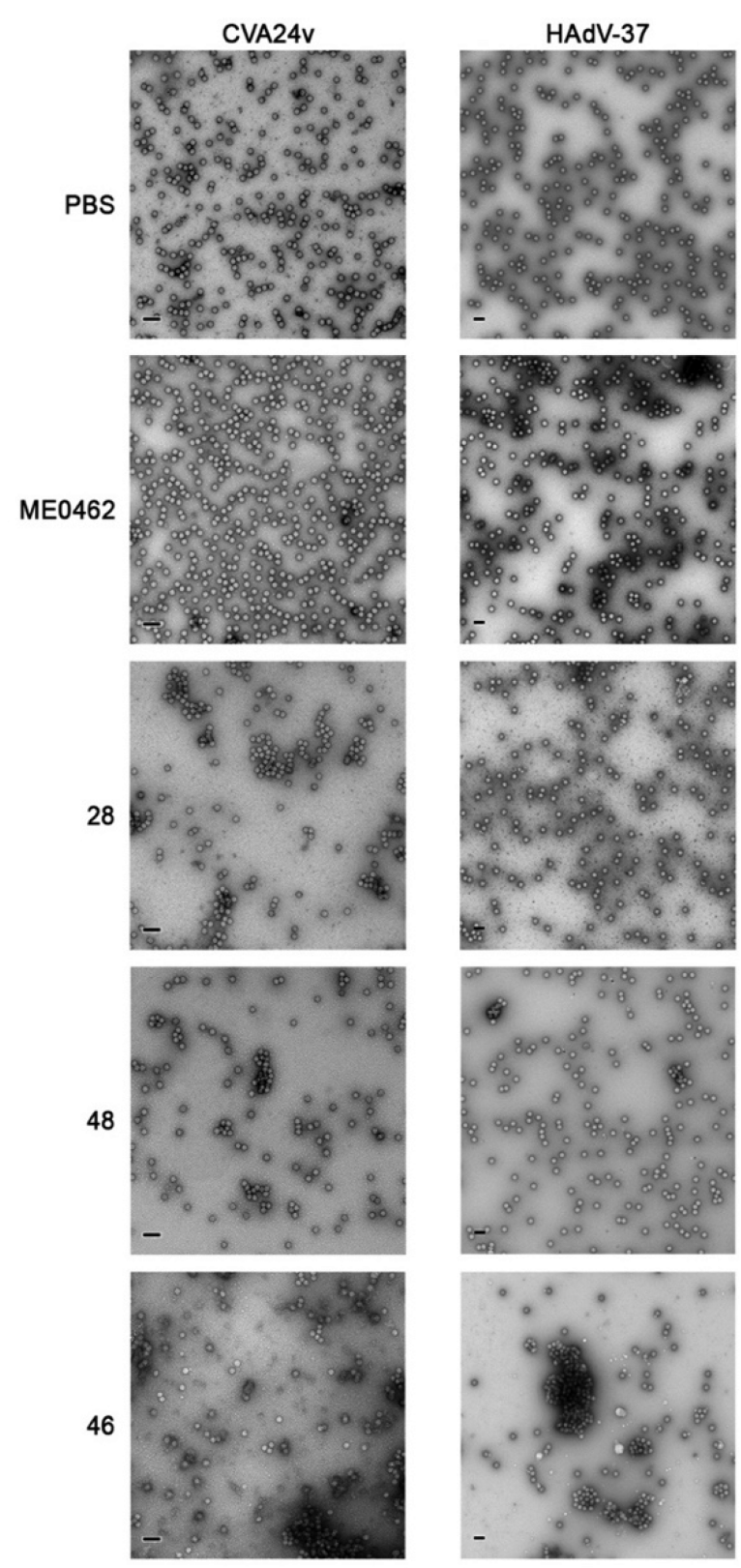

Figure 4. Effect of ME0462 and pentavalent sialic acid conjugates on cross-linking and aggregation of CVA24v and HAdV-37. The left panel shows the results of negative staining electron microscopy for CVA24v, and the right panel shows the results for HAdV-37 in PBS buffer or incubated with trivalent (ME0462) or pentavalent (28, 48, 46) sialic acid conjugates at a final concentration of compounds at 2 $\mathrm{mM}$. Scale bars are visible at the bottom left corner for each image and correspond to $100 \mathrm{~nm}$ for images with CVA24v and $200 \mathrm{~nm}$ for images with HAdV-37.

length and potency toward both CVA24v and HAdV-37. ME0462 was selected as a control as an aggregation mechanism seems unlikely due to the short spacers of this compound. Compared with untreated CVA24v (Figure 4, PBS left panel), ME0462 appears able to break up virion clusters present in the control, thus it does not cause aggregation or cross-linking of viral particles (Figure 4, ME0462 left panel).

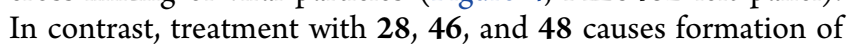
CVA24v aggregates. Moreover, the extent seems to be related to spacer length because larger aggregates were observed after treatment with 46 and 28 (long spacers) in comparison to 48
(Figure 4, 28, 46, and 48 of left panel). This indicates that aggregation may be the main mechanism of potency enhancement for the inhibitors in regards to CVA24v. It can, therefore, not be excluded that the sialic acid density observed in each of the five binding sites of the crystal structures originates from more than one pentavalent molecule crosslinking virus particles.

For HAdV-37, ME0462 showed very little to no aggregation or cross-linking of viral particles in comparison to untreated virions (Figure 4, PBS and ME0462 of right panel). Particles were also predominantly monodispersed after treatment with 28 and 48 (Figure 4, 28 and $\mathbf{4 8}$ of right panel). However, substantial aggregation and cross-linking were observed after treatment with 46 (Figure 4, 46 of right panel). Thus, it is reasonable to speculate that the main mechanism of potency enhancement for $\mathbf{2 8}$ and $\mathbf{4 8}$ occurs via a statistical rebinding mechanism by providing a high local concentration of sialic acid. This can also explain the slightly increased potency of $\mathbf{4 8}$ in comparison to $\mathbf{2 8}$ as shorter spacers provide a higher effective local concentration of ligands by a simple reasoning of distance between the unbound ligand and binding site. For 46, it appears that aggregation has major involvement in the mechanism of potency enhancement. In this particular system, aggregation does, however, not seem to cause additional enhancement in potency in comparison to a statistical rebinding mechanism.

In summary, we find it reasonable to speculate that the main mechanism of inhibition for these inhibitors involves binding to the virion thus blocking the virus from interacting with its cognate receptor(s) on the cellular surface. This inhibition mechanism can be complemented by, e.g., cross-linking of virus particles where the sialic acid residues and possibly the spacers in one compound bind to two virus particles.

The sialic acid binding sites of CVA24v and HAdV-37 are different. To further understand the binding of the inhibitors to CVA24v and HAdV-37 and the large differences in potency, we analyzed their respective binding sites. The sialic acid binding sites of each virus are located in the interface between two protomers of the biological assembly (pentamer and trimer, respectively). The sialic acid binding sites of HAdV-37 are more recessed and less solvent exposed because they are located on the inner side of the fiber knobs. They are also more rigid since there is less mobility in the loops interacting with sialic acid. ${ }^{33}$ In CVA24v, the receptorengaging loops show a higher degree of mobility and form very shallow binding sites that are solvent exposed. A comparison of the total buried surface area (BSA) per sialic acid entity does, however, show similar values of $160.5 \AA^{2}$ for CVA24v and $150.1 \AA^{2}$ for HAdV-37. In HAdV-37, the sialic acid moiety is recognized by direct contacts with Tyr312, Pro317, and Lys345 and via a water-mediated hydrogen bond to Ser344. ${ }^{33}$ A notable difference in the recognition is the presence of a salt bridge between the carboxylate group of sialic acid and Lys345. In the case of CVA24v, this electrostatic interaction is absent, and the carboxylate is hydrogen bonded to the amide of Ser147 and intramolecularly bonded to the C9 hydroxyl group of sialic acid (Figure 2D). To fully understand these differences in recognition and their potential contributions to inhibitor potency, further studies are required.

Another underlying factor to explain the potency difference could be that HAdV-37 exclusively relies on sialic acid glycans for attachment, while CVA24v engages both sialic acidcontaining glycans and the coreceptor ICAM-1. ${ }^{19}$ In summary, 
several factors seem to contribute in explaining the lower avidity of the pentavalent conjugates toward CVA24v.

\section{CONCLUSION}

In this study, we have presented the first inhibitors of CVA24v infection and demonstrated the inhibitors also effectively block HAdV-37 binding to HCE cells. Thus, the synthesized pentavalent sialic acid conjugates inhibit two of the major causative agents of AHC and EKC, supporting development of general inhibitors against these highly contagious eye infections. Topical administration directly to the target tissue in the eye circumvents many of the challenges facing a compound administered orally. The sialic acid binding sites are located on the virus surface, and the inhibitors, therefore, do not need to permeate any cell membranes, reducing the risk of off-target effects. Furthermore, systemic exposure is likely low reducing the risk for toxicity or other adverse effects originating from systemic distribution. General inhibitors are particularly attractive in this case due to clinical difficulties in distinguishing these infections and their causative agents. Such inhibitors could, therefore, be applied upon development of the first symptoms to alleviate the infections, limit the spread in the general population, and, most importantly, to help prevent health care-associated infections of immunocompromised individuals which can result in potentially life-threatening conditions.

\section{ASSOCIATED CONTENT}

\section{(s) Supporting Information}

The Supporting Information is available free of charge at https://pubs.acs.org/doi/10.1021/acschembio.0c00446.

Materials and methods, supplementary text on docking and calculations, Figures S1-S5, Tables S1-S3, chemical synthesis and analytical data, ${ }^{1} \mathrm{H}$ NMR and ${ }^{13} \mathrm{C}$ NMR spectra, and references (PDF)

\section{AUTHOR INFORMATION}

\section{Corresponding Author}

Mikael Elofsson - Department of Chemistry and Umeå Centre for Microbial Research, Umeå University, SE90187 Umeå, Sweden; (1) orcid.org/0000-0002-3219-4669; Email: mikael.elofsson@umu.se

\section{Authors}

Emil Johansson - Department of Chemistry, Umeå University, SE90187 Umeå, Sweden

Rémi Caraballo - Department of Chemistry, Umeå University, SE90187 Umeå, Sweden; ำ orcid.org/0000-0001-5912-8429

Nitesh Mistry - Section of Virology, Department of Clinical Microbiology, Umeå University, SE90185 Umeå, Sweden

Georg Zocher - Interfaculty Institute of Biochemistry, University of Tübingen, 72076 Tübingen, Germany

Weixing Qian - Department of Chemistry, Umeå University, SE90187 Umeå, Sweden

C. David Andersson - Department of Chemistry, Umeå University, SE90187 Umeå, Sweden

Daniel L. Hurdiss - Virology Division, Department of Infectious Diseases and Immunology, Faculty of Veterinary Medicine, Utrecht University, 3584CL Utrecht, The Netherlands

Naresh Chandra - Section of Virology, Department of Clinical Microbiology, Umeå University, SE90185 Umeå, Sweden
Rebecca Thompson - Astbury Centre for Structural Molecular Biology, Faculty of Biological Sciences, University of Leeds, Leeds LS2 9JT, United Kingdom

Lars Frängsmyr - Section of Virology, Department of Clinical Microbiology, Umeå University, SE90185 Umeå, Sweden

Thilo Stehle - Interfaculty Institute of Biochemistry, University of Tübingen, 72076 Tübingen, Germany; Vanderbilt University School of Medicine, Nashville, Tennessee 37232, United States

Niklas Arnberg - Section of Virology, Department of Clinical Microbiology, Umeå University, SE90185 Umeå, Sweden

Complete contact information is available at:

https://pubs.acs.org/10.1021/acschembio.0c00446

\section{Author Contributions}

E.J., R.C., and W.Q. synthesized all compounds. C.D.A. performed structure-based design. N.M. and N.C. evaluated the target compounds in cell-based assays. G.Z. performed the crystallography experiments. N.M. and L.F. carried out the thermal stability experiments. D.H.L. and R.T. carried out the electron microscopy studies of aggregation. E.J., R.C., M.E., N.A., and T.S. designed the experiments and analyzed the data together with all other authors. E.J. wrote most of the manuscript with contributions from all other authors. All authors have given approval to the final version of the manuscript.

Notes

The authors declare no competing financial interest.

\section{ACKNOWLEDGMENTS}

We thank the Diamond Light Source Ltd. (Didcot, UK) for beamtime and N. Paterson and M. Williams for their on-site support. The project was funded by a Knut and Alice Wallenberg Foundation grant (KAW; grant reference 2013.0019, to N.A. and M.E.) and by the Baden-Württemberg foundation, Glycobiology program (to T.S.).

\section{REFERENCES}

(1) Azari, A. A., and Barney, N. P. (2013) Conjunctivitis: a systematic review of diagnosis and treatment. JAMA 310, 1721-1729.

(2) Uchio, E. (2005) New medical treatment for viral conjunctivitis. Nippon Ganka Gakkai Zasshi 109, 962-984 discussion 985.

(3) Skevaki, C. L., Galani, I. E., Pararas, M. V., Giannopoulou, K. P., and Tsakris, A. (2011) Treatment of viral conjunctivitis with antiviral drugs. Drugs 71, 331-347.

(4) Centers for Disease Control and Prevention (2013) Adenovirusassociated epidemic keratoconjunctivitis outbreaks-four states, 20082010. MMWR 62, 637-641.

(5) Zhang, L., Zhao, N., Huang, X., Jin, X., Geng, X., Chan, T.-C., and Liu, S. (2017) Molecular epidemiology of acute hemorrhagic conjunctivitis caused by coxsackie A type 24 variant in China, 20042014. Sci. Rep. 7, 45202.

(6) Ghazali, O., Chua, K. B., Ng, K. P., Hooi, P. S., Pallansch, M. A., Oberste, M. S., Chua, K. H., and Mak, J. W. (2003) An outbreak of acute haemorrhagic conjunctivitis in Melaka, Malaysia. Singapore Med. J. 44, 511-516.

(7) Gonzalez, G., Yawata, N., Aoki, K., and Kitaichi, N. (2019) Challenges in management of epidemic keratoconjunctivitis with emerging recombinant human adenoviruses. J. Clin. Virol. 112, 1-9.

(8) Klinger, J. R., Sanchez, M. P., Curtin, L. A., Durkin, M., and Matyas, B. (1998) Multiple cases of life-threatening adenovirus pneumonia in a mental health care center. Am. J. Respir. Crit. Care Med. 157, 645-649.

(9) Huang, G., Yao, W., Yu, W., Mao, L., Sun, H., Yao, W., Tian, J., Wang, L., Bo, Z., Zhu, Z., Zhang, Y., Zhao, Z., and Xu, W. (2014) 
Outbreak of epidemic keratoconjunctivitis caused by human adenovirus type 56, China, 2012. PLoS One 9, e110781-e110781.

(10) Kurokawa, M., Rai, S. K., Ono, K., Gurung, R., and Ruit, S. (2006) Viral investigation of acute hemorrhagic conjunctivitis outbreak (2003) in Nepal using molecular methods. Southeast Asian J. Trop. Med. Public Health 37, 904-910.

(11) Wright, P. W., Strauss, G. H., and Langford, M. P. (1992) Acute hemorrhagic conjunctivitis. Am. Fam. Physician 45, 173-178.

(12) Oh, M.-d., Park, S., Choi, Y., Kim, H., Lee, K., Park, W., Yoo, Y., Kim, E.-C., and Choe, K. (2003) Acute hemorrhagic conjunctivitis caused by coxsackievirus A24 variant, South Korea, 2002,. Emerging Infect. Dis. 9, 1010-1012.

(13) Karnauchow, T. M., Tolson, D. L., Harrison, B. A., Altman, E., Lublin, D. M., and Dimock, K. (1996) The HeLa cell receptor for enterovirus 70 is decay-accelerating factor (CD55). J. Virol. 70, 51435152.

(14) Mistry, N., Inoue, H., Jamshidi, F., Storm, R. J., Oberste, M. S., and Arnberg, N. (2011) Coxsackievirus A24 variant uses sialic acidcontaining O-linked glycoconjugates as cellular receptors on human ocular cells. J. Virol. 85, 11283-11290.

(15) Nilsson, E. C., Jamshidi, F., Johansson, S. M. C., Oberste, M. S., and Arnberg, N. (2008) Sialic acid is a cellular receptor for coxsackievirus A24 variant, an emerging virus with pandemic potential. J. Virol. 82, 3061.

(16) Nokhbeh, M. R., Hazra, S., Alexander, D. A., Khan, A., McAllister, M., Suuronen, E. J., Griffith, M., and Dimock, K. (2005) Enterovirus 70 binds to different glycoconjugates containing alpha2,3linked sialic acid on different cell lines. J. Virol. 79, 7087-7094.

(17) Cifuente, J. O., and Moratorio, G. (2019) Evolutionary and structural overview of human picornavirus capsid antibody evasion. Front. Cell. Infect. Microbiol. 9, 283.

(18) Zocher, G., Mistry, N., Frank, M., Hähnlein-Schick, I., Ekström, J.-O., Arnberg, N., and Stehle, T. (2014) A sialic acid binding site in a human picornavirus. PLoS Pathog. 10, No. e1004401.

(19) Baggen, J., Hurdiss, D. L., Zocher, G., Mistry, N., Roberts, R. W., Slager, J. J., Guo, H., van Vliet, A. L. W., Wahedi, M., Benschop, K., Duizer, E., de Haan, C. A. M., de Vries, E., Casasnovas, J. M., de Groot, R. J., Arnberg, N., Stehle, T., Ranson, N. A., Thibaut, H. J., and van Kuppeveld, F. J. M. (2018) Role of enhanced receptor engagement in the evolution of a pandemic acute hemorrhagic conjunctivitis virus. Proc. Natl. Acad. Sci. U. S. A. 115, 397-402.

(20) Kaneko, H., Suzutani, T., Aoki, K., Kitaichi, N., Ishida, S., Ishiko, H., Ohashi, T., Okamoto, S., Nakagawa, H., Hinokuma, R., Asato, Y., Oniki, S., Hashimoto, T., Iida, T., and Ohno, S. (2011) Epidemiological and virological features of epidemic keratoconjunctivitis due to new human adenovirus type 54 in Japan. Br. J. Ophthalmol. 95, 32-36.

(21) Nilsson, E. C., Storm, R. J., Bauer, J., Johansson, S. M. C., Lookene, A., Ångström, J., Hedenström, M., Eriksson, T. L., Frängsmyr, L., Rinaldi, S., Willison, H. J., Domellöf, F. P., Stehle, T., and Arnberg, N. (2011) The GDla glycan is a cellular receptor for adenoviruses causing epidemic keratoconjunctivitis. Nat. Med. 17, $105-109$.

(22) Wu, E., Trauger, S. A., Pache, L., Mullen, T. M., von Seggern, D. J., Siuzdak, G., and Nemerow, G. R. (2004) Membrane cofactor protein is a receptor for adenoviruses associated with epidemic keratoconjunctivitis. J. Virol. 78, 3897-3905.

(23) Arnberg, N., Edlund, K., Kidd, A. H., and Wadell, G. (2000) Adenovirus type 37 uses sialic acid as a cellular receptor. J. Virol. 74, $42-48$.

(24) Varki, A. (2008) Sialic acids in human health and disease. Trends Mol. Med. 14, 351-360.

(25) Ernst, B., and Magnani, J. L. (2009) From carbohydrate leads to glycomimetic drugs. Nat. Rev. Drug Discovery 8, 661-677.

(26) Cecioni, S., Imberty, A., and Vidal, S. (2015) Glycomimetics versus multivalent glycoconjugates for the design of high affinity lectin ligands. Chem. Rev. 115, 525-561.

(27) Lundquist, J. J., and Toone, E. J. (2002) The cluster glycoside effect. Chem. Rev. 102, 555-578.
(28) Pieters, R. J. (2009) Maximising multivalency effects in proteincarbohydrate interactions. Org. Biomol. Chem. 7, 2013-2025.

(29) Kitov, P. I., Sadowska, J. M., Mulvey, G., Armstrong, G. D., Ling, H., Pannu, N. S., Read, R. J., and Bundle, D. R. (2000) Shigalike toxins are neutralized by tailored multivalent carbohydrate ligands. Nature 403, 669-672.

(30) Zhang, Z., Merritt, E. A., Ahn, M., Roach, C., Hou, Z., Verlinde, C. L. M. J., Hol, W. G. J., and Fan, E. (2002) Solution and crystallographic studies of branched multivalent ligands that Inhibit the receptor-binding of cholera toxin. J. Am. Chem. Soc. 124, 1299112998.

(31) Kitov, P. I., and Bundle, D. R. (2003) On the nature of the multivalency effect: A thermodynamic model. J. Am. Chem. Soc. 125, $16271-16284$.

(32) Spjut, S., Qian, W., Bauer, J., Storm, R., Frängsmyr, L., Stehle, T., Arnberg, N., and Elofsson, M. (2011) A potent trivalent sialic acid inhibitor of adenovirus type 37 infection of human corneal cells. Angew. Chem., Int. Ed. 50, 6519-6521.

(33) Caraballo, R., Saleeb, M., Bauer, J., Liaci, A. M., Chandra, N., Storm, R. J., Frangsmyr, L., Qian, W., Stehle, T., Arnberg, N., and Elofsson, M. (2015) Triazole linker-based trivalent sialic acid inhibitors of adenovirus type 37 infection of human corneal epithelial cells. Org. Biomol. Chem. 13, 9194-9205.

(34) Aplander, K., Marttila, M., Manner, S., Arnberg, N., Sterner, O., and Ellervik, U. (2011) Molecular wipes: application to epidemic keratoconjuctivitis. J. Med. Chem. 54, 6670-6675.

(35) Ortega-Muñoz, M., Morales-Sanfrutos, J., Perez-Balderas, F., Hernandez-Mateo, F., Giron-Gonzalez, M. a. D., Sevillano-Tripero, N., Salto-Gonzalez, R., and Santoyo-Gonzalez, F. (2007) Click multivalent neoglycoconjugates as synthetic activators in cell adhesion and stimulation of monocyte/machrophage cell lines,. Org. Biomol. Chem. 5, 2291-2301.

(36) Liese, S., and Netz, R. R. (2015) Influence of length and flexibility of spacers on the binding affinity of divalent ligands,. Beilstein J. Org. Chem. 11, 804-816.

(37) Marra, A., and Sinä̈, P. (1989) Stereoselective synthesis of 2thioglycosides of $\mathrm{N}$-acetylneuraminic acid. Carbohydr. Res. 187, 3542.

(38) Bornaghi, L. F., and Poulsen, S.-A. (2005) Microwaveaccelerated Fischer glycosylation. Tetrahedron Lett. 46, 3485-3488.

(39) Meijer, A., and Ellervik, U. (2002) Study of interhalogens/silver trifluoromethanesulfonate as promoter systems for high-yielding sialylations. J. Org. Chem. 67, 7407-7412.

(40) Tsang, S. K., Danthi, P., Chow, M., and Hogle, J. M. (2000) Stabilization of poliovirus by capsid-binding antiviral drugs is due to entropic effects. J. Mol. Biol. 296, 335-340.

(41) Martikainen, M., Salorinne, K., Lahtinen, T., Malola, S., Permi, P., Hakkinen, H., and Marjomaki, V. (2015) Hydrophobic pocket targeting probes for enteroviruses. Nanoscale 7, 17457-17467.

(42) Lacroix, C., Laconi, S., Angius, F., Coluccia, A., Silvestri, R., Pompei, R., Neyts, J., and Leyssen, P. (2015) In vitro characterisation of a pleconaril/pirodavir-like compound with potent activity against rhinoviruses. Virol. J. 12, 106-106.

(43) Liu, Y., Sheng, J., Fokine, A., Meng, G., Shin, W.-H., Long, F., Kuhn, R. J., Kihara, D., and Rossmann, M. G. (2015) Structure and inhibition of EV-D68, a virus that causes respiratory illness in children. Science 347, 71-74.

(44) Walter, T. S., Ren, J., Tuthill, T. J., Rowlands, D. J., Stuart, D. I., and Fry, E. E. (2012) A plate-based high-throughput assay for virus stability and vaccine formulation. J. Virol. Methods 185, 166-170. 